\begin{tabular}{|c|c|}
\hline Title & Multi-Objective Topology Optimization of Rotating Machines U sing Deep Learning \\
\hline Author(s) & Doi, Shuhei; Sasaki, Hidenori; Igarashi, Hajime \\
\hline Citation & $\begin{array}{l}\text { IEEE transactions on magnetics, 55(6), } 7202605 \\
\text { https://doi.org/10.1109/T MA G.2019.2899934 }\end{array}$ \\
\hline Issue Date & 2019-06 \\
\hline Doc URL & http://hdl.handle.net/2115/74691 \\
\hline Rights & $\begin{array}{l}\text { (c) } 2019 \text { IEEE. Personal use of this material is permitted. Permission from IEEE must be obtained for all other uses, in } \\
\text { any current or future media, including reprinting/republishing this material for advertising or promotional purposes, } \\
\text { creating new collective works, for resale or redistribution to servers or lists, or reuse of any copyrighted component of } \\
\text { this work in other works. }\end{array}$ \\
\hline Tyре & article (author version) \\
\hline File Information & Full paper final submission.pdf \\
\hline
\end{tabular}

Instructions for use 


\title{
Multi-Objective Topology Optimization of Rotating Machines Using Deep Learning
}

\author{
Shuhei Doi ${ }^{1}$, Hidenori Sasaki ${ }^{1}$, and Hajime Igarashi ${ }^{1}$ \\ ${ }^{1}$ Graduate School of Information Science and Technology, Hokkaido University, Sapporo, 060-0814, Japan
}

\begin{abstract}
This paper presents fast topology optimization methods for rotating machines based on deep learning. The cross-sectional image of electric motors and their performances obtained during a multi-objective topology optimization based on the finite element method (FEM) and genetic algorithm (GA) are used for training of convolutional neural network (CNN). Two different approaches are proposed: (i) CNN trained by preliminary optimization with small population for GA is used for main optimization with large population, (ii) CNN is used for screening of torque performances in the optimization with respect to the motor efficiency.
\end{abstract}

Index Terms - Deep learning, Genetic algorithm, IPM motor, Multi-objective optimization, Topology optimization.

\section{INTRODUCTION}

$\mathrm{R}_{\mathrm{e}}^{\mathrm{e}}$ ecently, the effective design method for electric motors especially for electric vehicles has been strongly required. In optimization of electric motors, it is necessary to consider many properties such as average torque, torque ripple, iron loss, radial force and so on. The multi-objective optimization based on stochastic algorithm, e.g., genetic algorithm (GA), allows us to obtain Pareto solutions with respect to multiple motor properties. By increasing the population and children sizes of GA, one would obtain the sufficiently populated Pareto solutions. The optimization based on GA with rich population, however, needs large computational cost due to a number of field computations with e.g. finite element method (FEM). Thus, solution of such multi-objective problems would lead to unallowable computational cost.

Deep learning (DL) based on convolutional neural network (CNN) has been shown effective for fast evaluation of the performance of an electric motor [1] and also acceleration of the topology optimization [1]. In order to reduce the number of evaluations by FEM in the multi-objective optimizations, we propose here an optimization method based on DL. In this method, the cross-sectional images of electric motors and their performance obtained by a topology optimization is used for training of CNN. Then, $\mathrm{CNN}$ is used as the surrogate method of FEM for acceleration of the multi-objective topology optimization. Two different approaches are proposed here: (i) CNN trained by a preliminary optimization with small population of GA is used for main optimization with large population, (ii) CNN trained by an optimization with respect to torque performances is used for screening of the torque performances in another optimization with respect to the motor efficiency. The first approach is effective to obtain a sufficiently populated Pareto solution using GA with rich population. The second approach can be generalized as follows:

Manuscript received April 1, 2015; revised May 15, 2015 and June 1, 2015; accepted July 1, 2015. Date of publication July 10, 2015; date of current version July 31, 2015. (Dates will be inserted by IEEE; "published" is the date

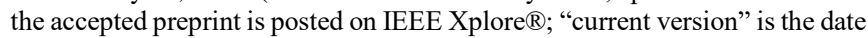
the typeset version is posted on Xplore $\left.{ }^{\circledR}\right)$. Corresponding author: S.Doi (email: doi@em.ist.hokudai.ac.jp).

gures in this paper are available online at http://ieeexplore.ieee.org.

Digital Object Identifier (inserted by IEEE).
CNN is trained through data obtained by solving problem A, then the trained $\mathrm{CNN}$ is extendedly used for fast optimization of problems B, C... with different cost functions or constraints. These approaches will be verified by numerical examples.

\section{Multi-OBJective Topology OptimiZATION WITH CNN}

\section{A. Why deep learning}

In this work, the data composed of the cross-sectional images of electric motors and their performance obtained during the topology optimization process are used for training of $\mathrm{CNN}$, which is then used to reduce the field computing cost of optimization with other cost functions, constraints and models. There exist several approximate computing methods for optimization that are used for evaluation of fitness instead of time-consuming field computations using, e.g. FEM. They include the response surfaces [3][4], kriging method[5], artificial neural network (ANN) [6]. Although the former two methods are effective when degree of freedoms (DoFs) is less than, say, ten, DoFs in the pixel image of an electric motor is far larger. ANN can treat the images, while the image features for accurate classification have to be extracted by the user. However, it would be hard to extract such features from diverse images generated through the topology optimization. In contrast, $\mathrm{CNN}$ can automatically extract such features from the training images. For these reasons, we employ $\mathrm{CNN}$ for the surrogate model in this work.

\section{B. Classifier using $C N N$}

In the learning phase, we construct the classifiers of the average torque $T_{\text {ave }}$ and torque ripple $T_{\text {rip }}=$ $\left(T_{\max }-T_{\min }\right) / T_{\text {ave }}$ using CNN with 22 layers, for which GoogLenet is employed [7]. The structure of $\mathrm{CNN}$ is schematically shown in Fig. 1. The input data for CNN is a bitmap image representing the cross-sectional shape of an inner permanent magnet (IPM) motor. The output data is the corresponding values of $T_{\text {ave }}$ or $T_{\text {rip }}$. The classes provided by CNN are summarized in TABLE I, TABLE II where $T_{\mathrm{ave}}^{\mathrm{FEM}}, T_{\text {rip }}^{\mathrm{FEM}}$ denote the average torque and torque ripple computed with FEM, while $T_{\text {ave }}^{\mathrm{CNN}}, T_{\text {rip }}^{\mathrm{CNN}}$ denote the classes output from $\mathrm{CNN}$. The training data is obtained by a 
preliminary multi-objective topology optimization of the IPM motor shown in Fig. 2 with relatively small populations in GA. In the topology optimization, the element state $S_{e}$ is determined from the shape function $\varphi(\boldsymbol{x})=\sum_{i}^{n} w_{i} G_{i}(\boldsymbol{x})$ where $G_{i}$ and $w_{i}$ denote the normalized Gaussian function and weighting coefficient such that $S_{e}=$ iron (air) if $\varphi \geq 0(<0)$ [8]. The optimization problem is defined as follows:

$$
\max T_{\text {ave }}(\boldsymbol{w}), \min T_{\text {rip }}(\boldsymbol{w})
$$

where $\boldsymbol{w}$ denotes the vector composed of $w_{i}, i=1,2, \ldots, n$. Note that the material distribution and thus machine performance depend on $\boldsymbol{w}$. The population sizes are summarized in the left column of TABLE III. Fig. 3 shows the distribution of $T_{\mathrm{ave}}^{\mathrm{CNN}}$ in the initial population whose individuals are randomly generated. As shown in Fig. 3, many individuals belong to the classes with low average torques. Such individuals could be approximately evaluated only by $\mathrm{CNN}$ because these have little influence on the evolution. On the other hand, individuals in classes with higher torques should be accurately evaluated by FEM. Namely, the trained CNN can be effectively used for screening of the torque performances.

We use 6000 data obtained during the multi-objective optimization of the IPM motor with respect to $T_{\text {ave }}, T_{\text {rip }}$ using GA with the size parameters in the left column of Table III for the training of CNN. The 700 data that are not used for the training are used for test of CNN. TABLE IV, TABLE V summarizes the classification results. It is found that there are small number of off-diagonal samples. The reason why the classification accuracy in $T_{\text {rip }}$ is lower than that in $T_{\text {ave }}$ is due to the relatively weak correlation between the material distribution and torque ripple.

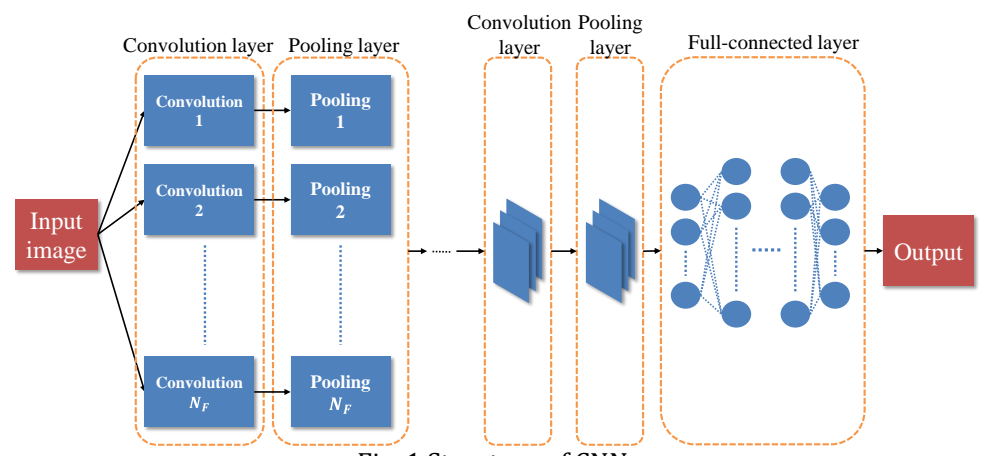

Fig. 1 Structure of CNN

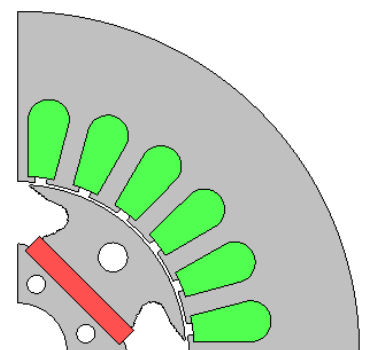

Fig. 2. IPM motor obtained by preliminary optimization

\section{Optimization Method (i)}

We use the CNN trained for the data obtained through the

preliminary optimization process mentioned above for the acceleration of the main multi-objective topology optimization with larger population size, which is summarized in the center column of TABLE III.

In the main multi-objective optimization process after the learning phase, the values $T_{\mathrm{ave}}^{\mathrm{CNN}}, T_{\text {rip }}^{\mathrm{CNN}}$ are evaluated by the trained $\mathrm{CNN}$ to make fast evaluation of the rank $F_{i}^{\mathrm{CNN}} \in \mathbb{Z}$ of individual $i$ using non-dominated sorting by NSGA-II [9]. Since there would be errors in the ranking, the correction is made by performing finite element (FE) analysis with probability $P_{i}$ given by

$$
P_{i}=\left(\frac{F_{\mathrm{worst}}^{\mathrm{CNN}}-F_{i}^{\mathrm{CNN}}}{F_{\mathrm{worst}}^{\mathrm{CNN}}-1}\right)^{2}
$$

where $P_{i}$ is evaluated over the whole population in the first generation and over the children in other generations. We design $P_{i}$ so that it increases as the score provided by CNN becomes better. In Fig. 5, $P_{i}$ is plotted against $F_{i}^{\mathrm{CNN}}$. In the first steps of GA, a limited number of individuals would be analyzed by FEM because the majority of population tends to

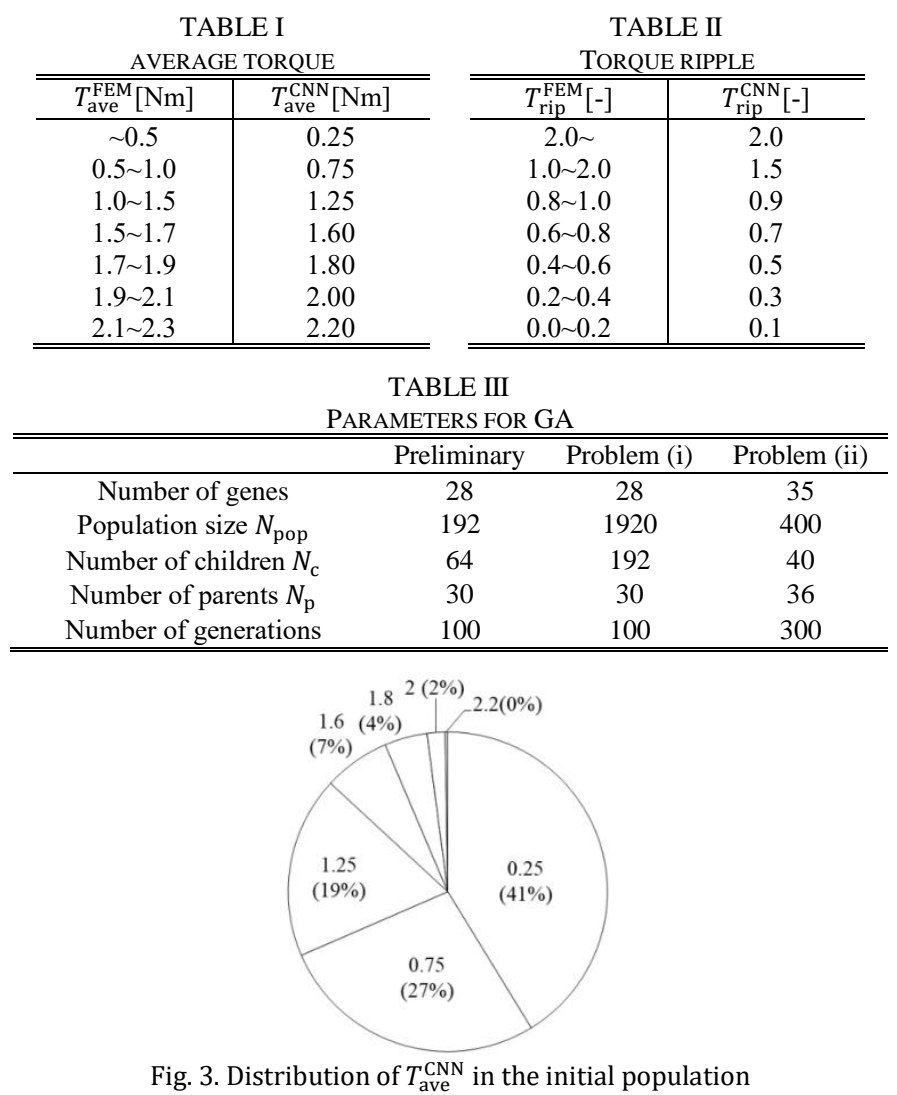

\begin{tabular}{|c|c|c|c|c|c|c|c|c|}
\hline $\mathrm{CL}$ & SI & FIEF & OF & $\mathrm{AVE}$ & & & $Q$ & \\
\hline Accl & acy & & & & $\mathrm{NN}$ & & & \\
\hline 92 & & 0.25 & 0.75 & 1.25 & 1.6 & 1.8 & 2 & \\
\hline & $\overline{0.25}$ & 53 & $\overline{2}$ & $\overline{0}$ & $\overline{0}$ & $\overline{0}$ & $\overline{0}$ & \\
\hline & 0.75 & 0 & 83 & 8 & 0 & 0 & 0 & \\
\hline & 1.25 & 0 & 4 & 87 & 5 & 0 & 0 & \\
\hline FEM & 1.6 & 0 & 0 & 6 & 108 & 5 & 0 & \\
\hline & 1.8 & 0 & 0 & 0 & 7 & 102 & 5 & \\
\hline & 2 & 0 & 0 & 0 & 0 & 6 & 117 & 3 \\
\hline & 2.2 & 0 & 0 & 0 & 0 & 0 & 2 & 97 \\
\hline
\end{tabular}

TABLE IV

have low values of $F_{i}^{\mathrm{CNN}}$. The algorithm of the main multiobjective optimization is shown in Fig. 6.

\begin{tabular}{|c|c|c|c|c|c|c|c|}
\hline \multirow{2}{*}{$\begin{array}{c}\text { CLAS } \\
\text { Accuracy } \\
81 \%\end{array}$} & \multicolumn{7}{|c|}{$\mathrm{CNN}$} \\
\hline & 0.1 & 0.3 & 0.5 & 0.7 & 0.9 & 1.5 & 2 \\
\hline \multirow{7}{*}{ FEM } & 82 & 5 & $\overline{0}$ & $\overline{0}$ & $\overline{0}$ & $\overline{0}$ & 0 \\
\hline & 9 & 71 & 8 & 4 & 0 & 0 & 0 \\
\hline & 0 & 14 & 78 & 9 & 1 & 0 & 0 \\
\hline & 0 & 0 & 8 & 94 & 5 & 1 & 0 \\
\hline & 0 & 3 & 1 & 15 & 84 & 13 & 1 \\
\hline & 0 & 2 & 1 & 1 & 15 & 82 & 7 \\
\hline & 0 & 0 & 0 & 0 & 0 & 8 & 78 \\
\hline
\end{tabular}

TABLE V

.




\section{Optimization Method (ii)}

We consider here the optimization to reduce the iron loss of the IPM motor keeping the torque performance better than a threshold. The optimization model is shown in Fig. 4. To make the computing cost smaller, CNN trained for the preliminary optimization is used for screening of the torque performances. The optimization problem is defined by

$$
\min W_{\text {iron }}, \quad \text { sub. to } T_{\text {ave }}>2.0 \text { AND } T_{\text {rip }}<0.2
$$

where $W_{\text {iron }}$ denotes the iron loss, which is here evaluated by the Steinmetz formula

$$
W_{\text {iron }}=k_{h} f\left(\frac{B_{\mathrm{a}}}{B_{0}}\right)^{2}+k_{e} f^{2}\left(\frac{B_{\mathrm{a}}}{B_{0}}\right)^{2}
$$

where $B_{\mathrm{a}}$ and $f$ denotes the maximum amplitude and frequency of the magnetic flux density, and the constants are set as follows: $B_{0}=1, k_{h}=7.5 \times 10^{-2} \mathrm{~J}, k_{e}=6.0 \times 10^{-4} \mathrm{Js}$.

In this optimization, we evaluate $T_{\text {ave }}, T_{\text {rip }}$ using the trained $\mathrm{CNN}$, and if they satisfy the prescribed condition

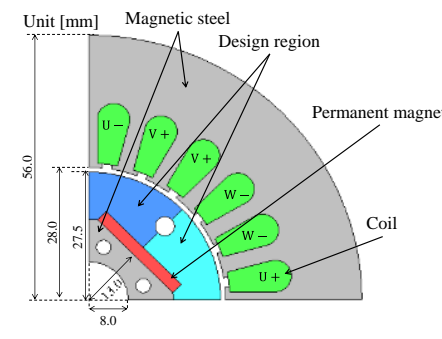

Fig. 4. Optimization model

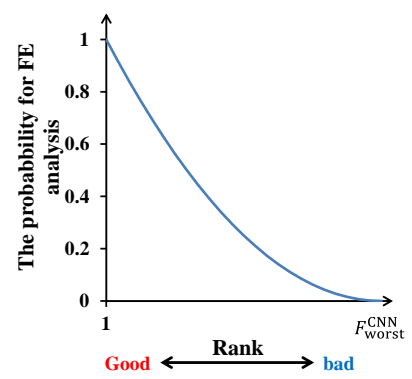

Fig. 5. Probability $P_{i}$ for execution of FE analysis

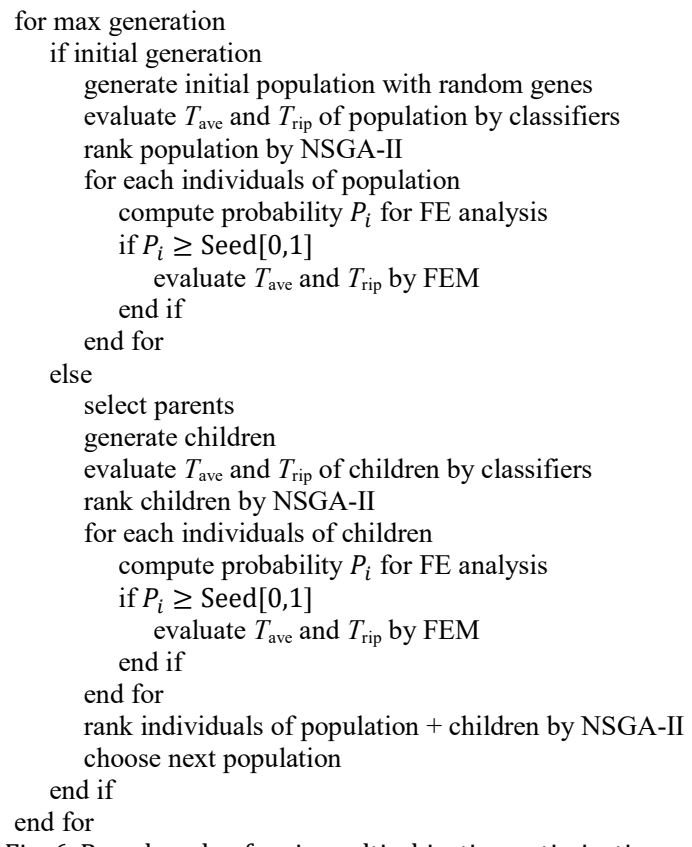

$$
T_{\text {ave }} \geq 1.7 \text { AND } T_{\text {rip }} \leq 0.4
$$

We impose this weakened condition for FE analysis because the original constraint in (3) is too strict to have sufficiently large number of population that evolves toward the optimal solution. In the first steps of the GA process, the majority of population does not satisfy (5). Thus, the number of FE analysis is strongly suppressed. The GA setting is summarized in the right column of TABLE III.

\section{OPTIMIZATION RESULTS}

\section{A. Result Obtained by Method (i)}

Fig. 8 shows the Pareto solutions at the 100th generation of GA obtained by the preliminary and main multi-objective optimizations. Using the proposed method, the Pareto solutions can be refined with reduced computational cost. The number of FE analysis, normalized by the total number of ranking evaluation, is plotted against the GA generations in Fig. 9. Because the individuals with higher ranking increase with the generation, the number of $\mathrm{FE}$ evaluations increases. In the entire optimization process, FE analysis is performed for $49.5 \%$ of the individuals.

The shapes of the IPM motors on the Pareto front, marked by A and B in Fig. 8, are shown in Figs 10. and 11, where solution A has the largest average torque but large torque ripple, while $\mathrm{B}$ has a good balance in these two torque performances. The rotor of solution A has large notches which would yield large reluctance torque as well as large torque ripple. On the other hand, the rotor of solution B has a smooth surface, hence has a lower torque ripple and average torque.

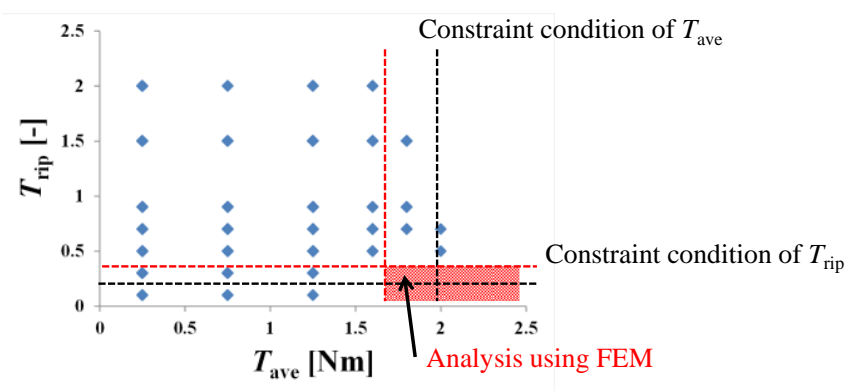

Fig. 7. Domain for execution of FE analysis

- Preliminary optimization $\diamond$ Main optimization

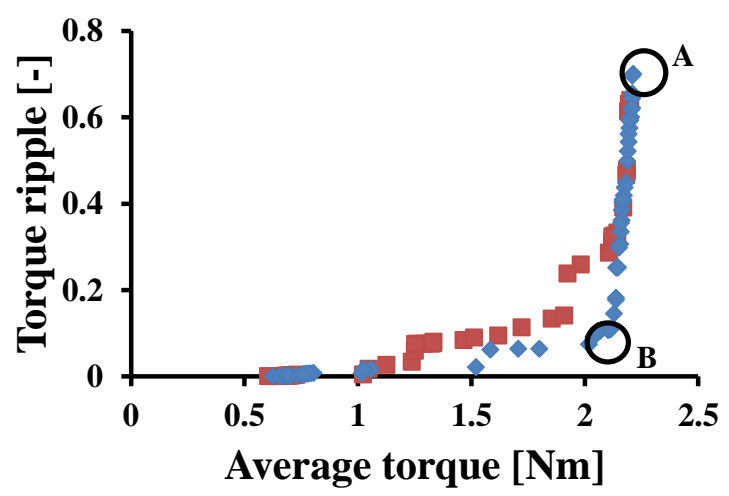

Fig. 8. Comparison of Pareto solutions obtained by method (i)

these torque properties as well as iron loss are evaluated by FEM. The region satisfying this condition is shown in Fig. 7. 


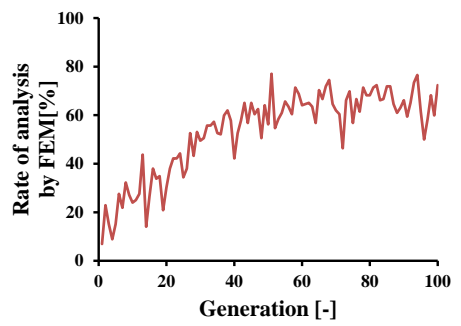

Fig. 9. Rate of FEM evaluations

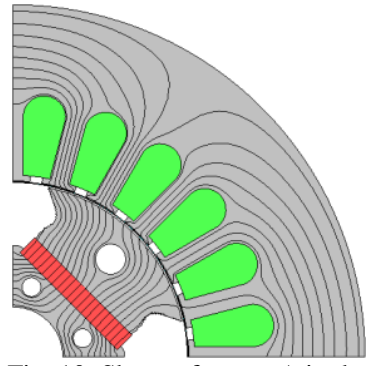

Fig. 10. Shape of motor A in the pareto front shown in Fig. 8 $T_{\text {ave }}=2.21 \mathrm{Nm}, T_{\text {rip }}=0.700$

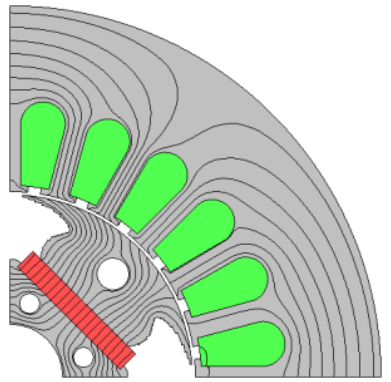
obtained by method (ii). $W_{\text {iron }}=1.75 \times 10^{-3}$, $T_{\text {ave }}=2.00 \mathrm{Nm}, T_{\text {rip }}=0.191$
Fig. 12. Optimization result

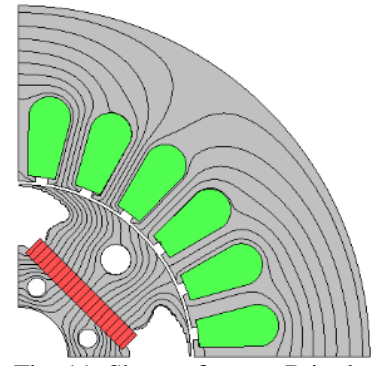

Fig. 11. Shape of motor B in the pareto front shown in Fig.8 $T_{\text {ave }}=2.02 \mathrm{Nm}, T_{\text {rip }}=0.074$

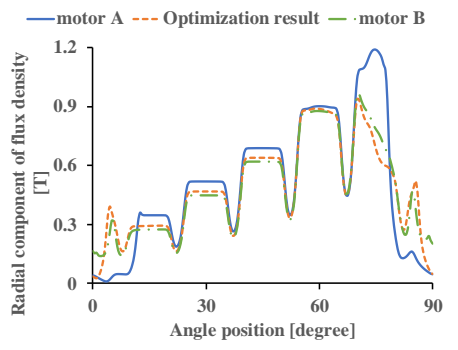

Fig. 13. Radial component of flux density along the midline of the air-gap

\section{B. Result Obtained by Method (ii)}

Fig. 12 shows the optimization result, which is found to meet the constraints. The flux distribution in the air gap is plotted in Fig.13. The iron loss, average torque and torque ripple of the best individual are plotted against the generation of GA in Fig. 14(a)-(c). The iron loss is not plotted until 21th generation because no individuals are analyzed by FEM. The torque ripple goes up and down because the optimization seeks for a ground for compromise between the average torque and torque ripple which have a reciprocal relationship.

The number of FE analysis, normalized by the total number of ranking evaluation, is plotted against the generation of GA in Fig. 14(d). In the early stage of optimization, the number of FE analysis is suppressed, as expected, because only small number of population satisfies condition (5). As evolution progresses, the execution rate of the FE analysis grows because individuals satisfying (5) increase. As a result, the number of $\mathrm{FE}$ analysis is reduced to approximately $30 \%$. This rate depends on the condition for the FE analysis. If we make (5) stricter, then the number of FE analysis would be further suppressed. However, the evolution would not be successful when the condition is made too strict because the number of individuals satisfying such condition is limited.

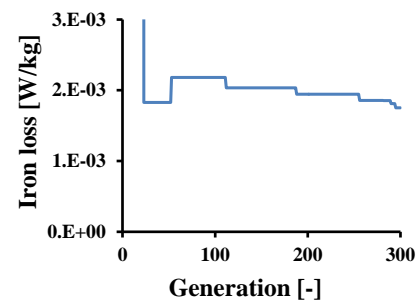

(a) Evolution of the best $W_{\text {iron }}$

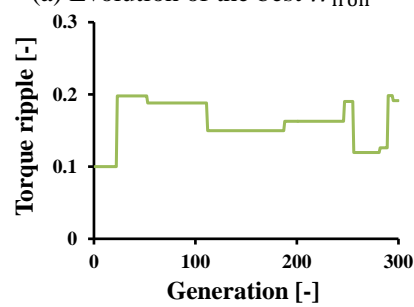

(c) Evolution of the best $T_{\text {rip }}$

Fig. 14. Evolution of the best valu

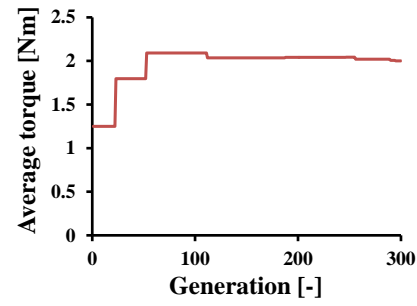

(b) Evolution of the best $T_{\text {ave }}$

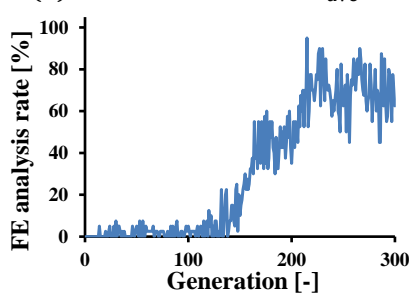

(d) Change of the analysis rate

\section{CONCLUSION}

In this paper, we have proposed new methods based on DL to accelerate the multi-objective topology optimization. In method (i), the CNN trained through the preliminary optimization with small population is used to make a fast evaluation of fitness in the main optimization with large population. In method (ii), the CNN trained through the preliminary optimization with respect to the torque performances is used for fast evaluation of constraints in the optimization with respect to iron loss. The FE computations are reduced to about $50 \%$ and $30 \%$, respectively, by (i) and (ii).

We plan to study methods for adequate design of the probability function and the condition for the FE analysis. We will also study adequate modification of the shape obtained by the topology optimization for industrial realization.

\section{REFERENCES}

[1] H. Sasaki, H. Igarashi, "Topology optimization of IPM motor with aid of deep learning", to be published in Int. J. Appl. Electromagnetics and Mech.

[2] H. Sasaki, H. Igarashi, "Topology Optimization Accelerated by Deep Learning", to be published in IEEE Trans. Magn.,

[3] R. H. Myers, D. C. Montgomery, and C. M. Anderson-Cook, "Response surface methodology : Process and product optimization using designed experiments" 3rd Ed., Wiley, 2009.

[4] Y. Duan, D.M. Ionel, "A Review of Recent Developments in Electrical Machine Design Optimization Methods With a Permanent-Magnet Synchronous Motor Benchmark Study," IEEE Trans. Industry App. 49(3), 1268-1275, 2013.

[5] S. Xiao, et al., "Adaptive Weighted Expected Improvement With Rewards Approach in Kriging Assisted Electromagnetic Design, "IEEE Trans. Magn., vol. 49, no. 5, 2057-2060, 2013.

[6] S. Shimokawa, et al. "Fast 3-D Optimization of Magnetic Cores for Loss and Volume Reduction", IEEE Trans. Magn., vol. 54, no. 11, 8400904, 2018.

[7] C. Szegedy, et al. "Going deeper with convolutions." Proceedings of the IEEE conference on computer vision and pattern recognition, 2015.

[8] T. Sato, K. Watanabe, H. Igarashi, "Multimaterial Topology Optimization of Electric Machines Based on Normalized Gaussian Network", IEEE Trans. Magn., vol. 51, no. 3, 7202604, 2015.

[9] K. Deb, A. Pratap, S. Agarwal, and T. Meyarivan. A fast and elitist multiobjective genetic algorithm: NSGA-II. IEEE Trans. On evolutionary Computation, 6(2):182-197, 2002. 